\title{
تقدير الدالة المعولية للانظمة متعدد الحالات بأستخدام المشتقة الجزئية المنطقية المباشرة
}

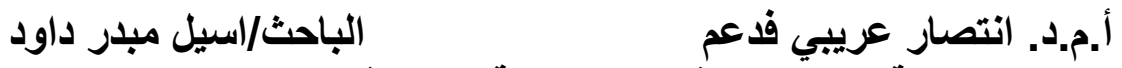 \\ كلية الادارة والاقتصاد/ جامعة بغداد/ قسم الاحصاء
}

\begin{abstract}
المستخلص من
في هذا البحث يتم تقدير المعولية الديناميكية للنظام المتعدد الحالة ولكل مكون من مكوناته ولثثلاثة

انواع من الانظمة (المتسلسل، المتوازي ،

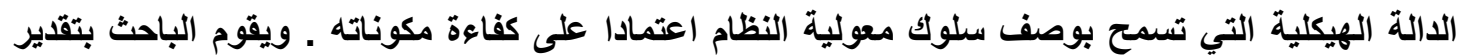

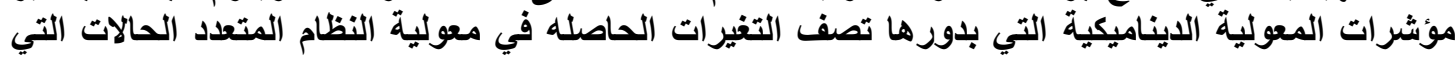

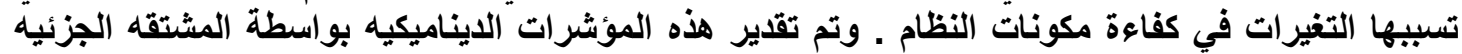

المنطقيه المباثرة

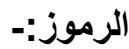

Mss - : النظام المتعدد الحالات

MSSRF

SFOMSS

DRI

مجموعة حالات الفشل لكل مكون : : : Gf / xi \}

مجموعة حالات الاصلاح لكل مكون :- : : Gf / Xi \}

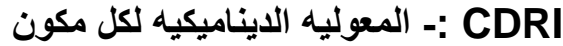

(المعوليه الديناميكيه للنظام باكمله :- DIRI

$\varphi(x)$
\end{abstract}

mball

|

المجلد 20

العدد 7

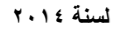

الصفحات333 -350 


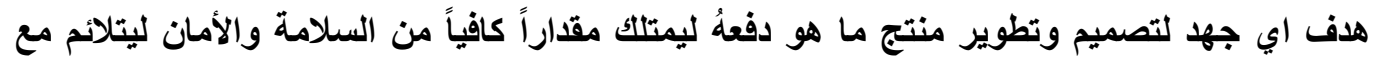

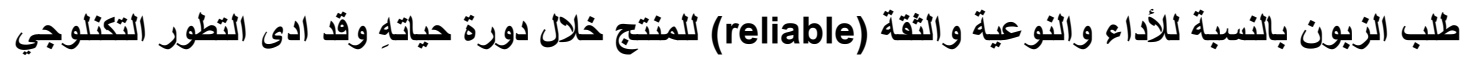

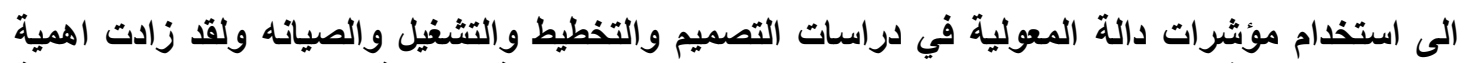

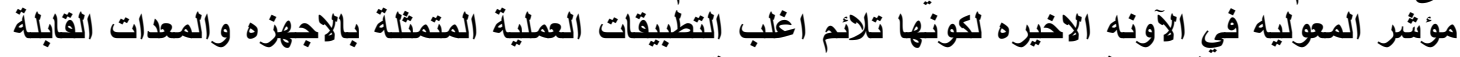

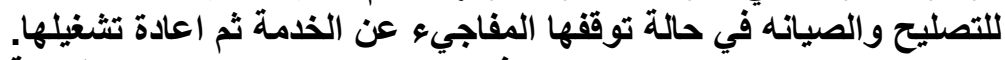

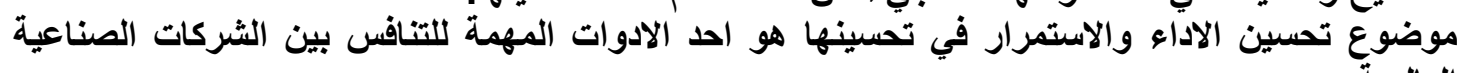

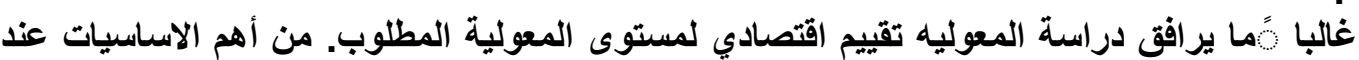

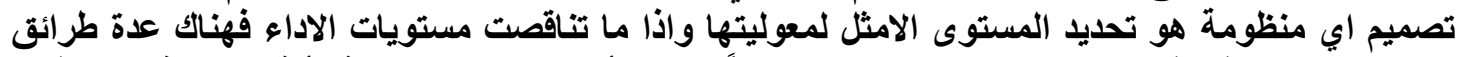

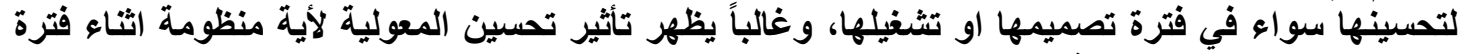
التثغيل لها من خلأل الصيانة. ولتحليل المعولية هناك نموذجين رياضين المين الاول هول هو النظام الثنائي الذي يسمح للنظام ومكوناته

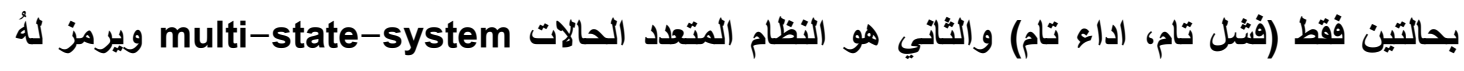

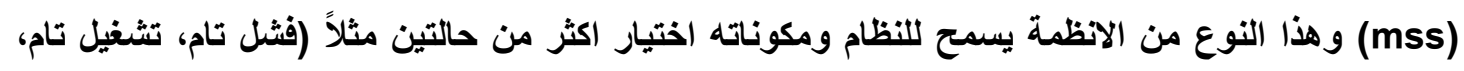
تشغيل جزئي). في الواقع النظام الثنائي هو ابسط حاله للأنظمه المتعددة الحالات لانه يمثل حالتين فقط.

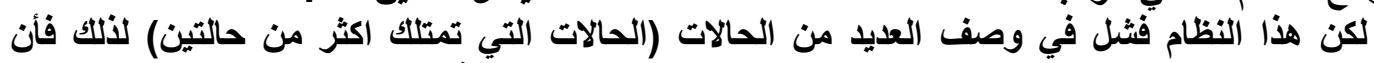

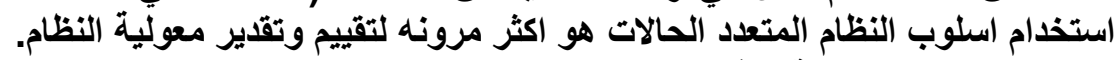

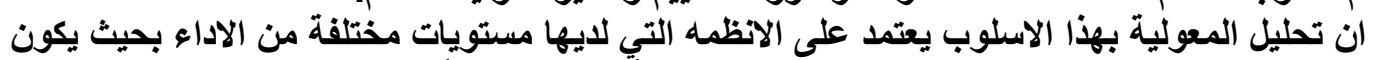

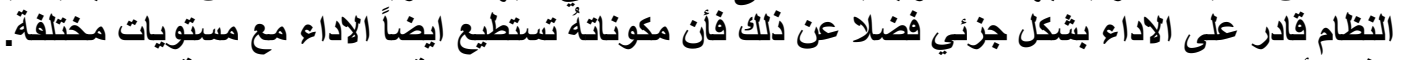

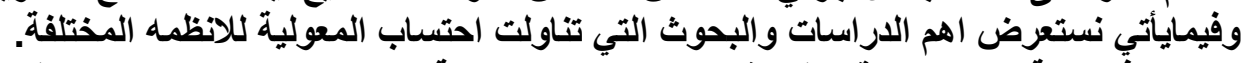

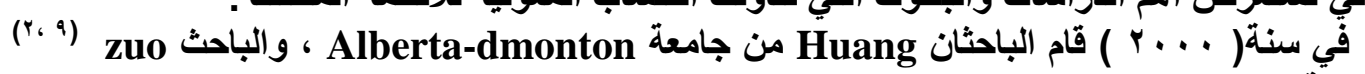

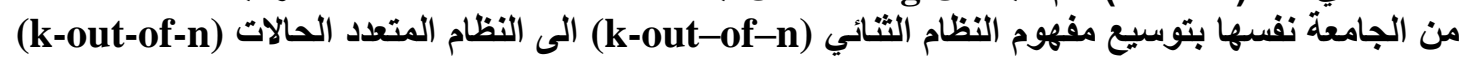
ولكن مع وجود قيود فمن المفترض ان يكون للثابت k مستويات في النظام الكلي .

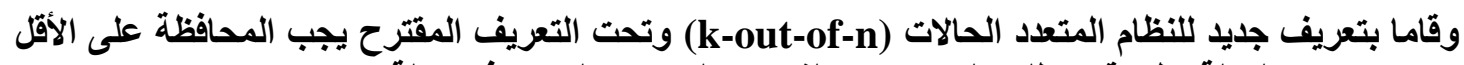

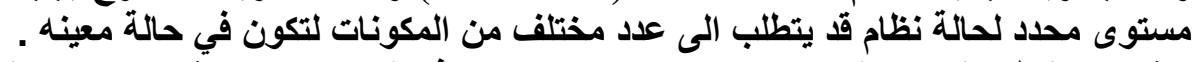
و اثبتا ان النظام المتعدد الحالات (k-out-of-n) هو نموذج له فن خصائص اكثر تعقيدا من النظام الثنائي . (k-out-of-n )

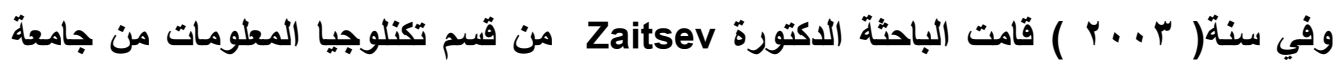

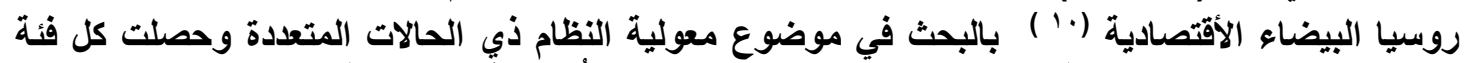

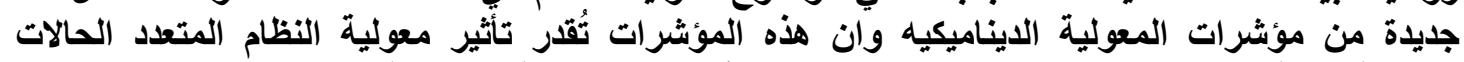

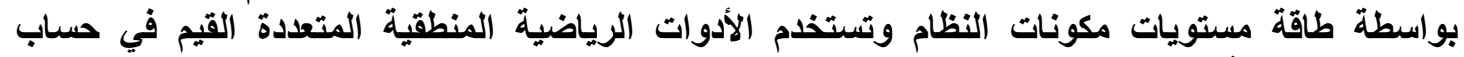

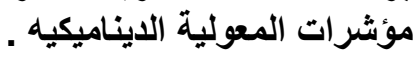

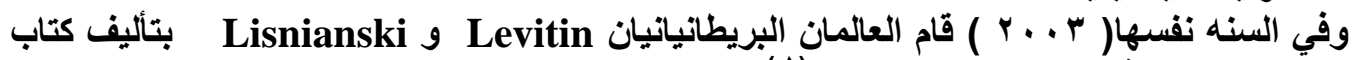

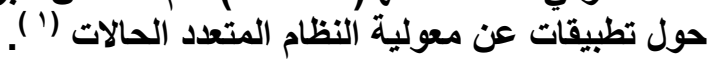
ويشير الكتاب الى طرائق تقدير معولية الانظمة المتعددة الحالات وتطوير هذه الطر ائق وتطبيقاتها. 


\section{المنطقية المباشرة}

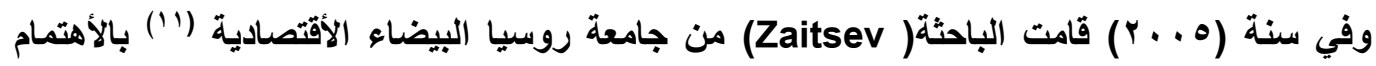

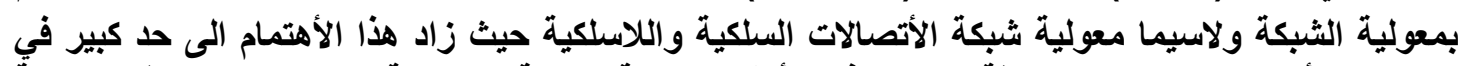

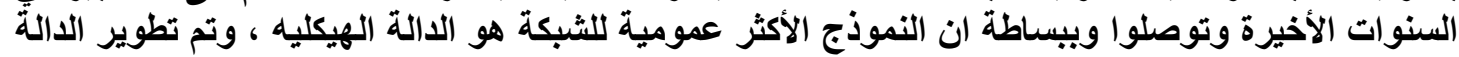

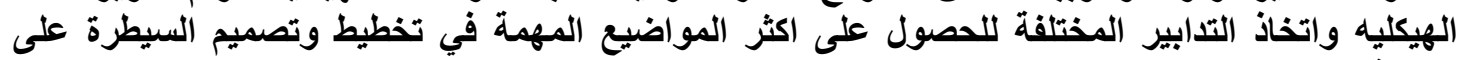

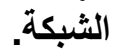

في سنة(1995) اقترح الباحثان( Xue و Yang ) الجمع بين عمليات ماركوف والدالة الهيكليه لتقاير

الخصائص الايناميكيه للأنظمة المتعددة الحالات (3) واثبتا ايضا ان منهجية الدالة تسمح بتحليل التغيرات

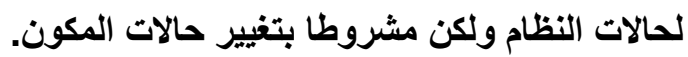

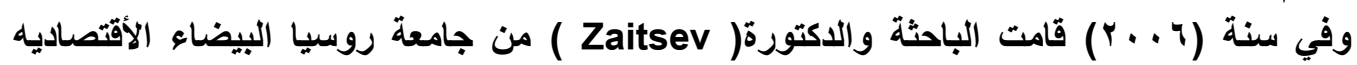

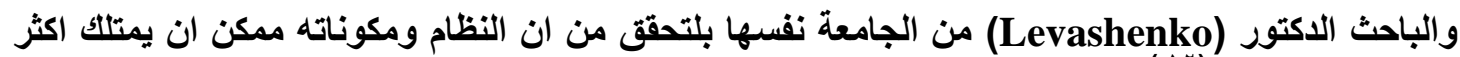

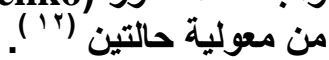
فقاما بأثبات ان مؤشرات المعولية الديناميكيه (DRI) تُفسر على انها احتمالات التغييرات في معولية النظام

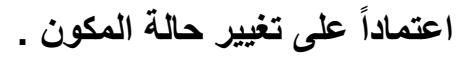

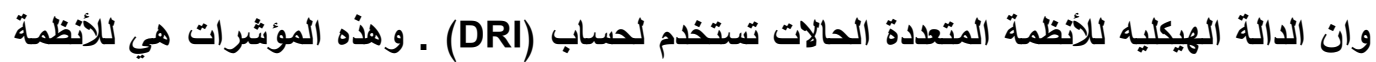

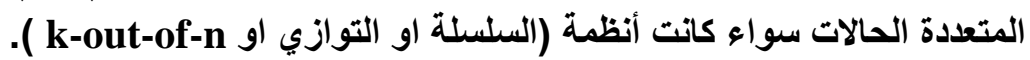

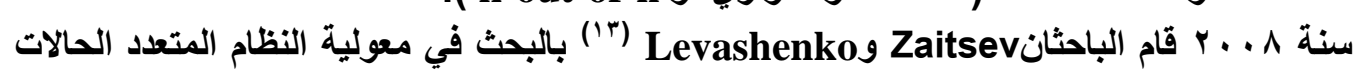

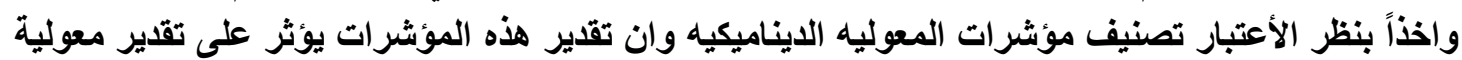

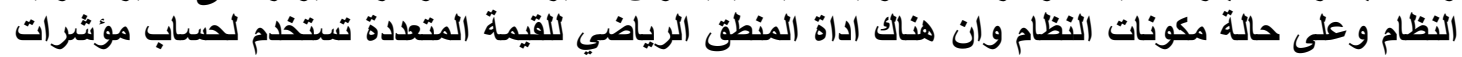

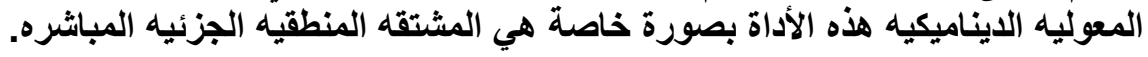

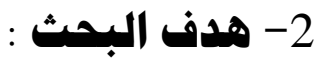

تقدير دالة المعولية للمركبه وللأنظمة المتعددة الحالات (المتسلسلة والمتوازية و (2-out-of-3)

ولحالتين (الفشل، والاصلاح) (failure, repear) Rf, المباشرة للارالة الهيكلية.(Direct Partial Logic Derivative for structure function)

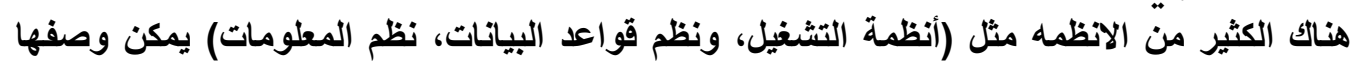

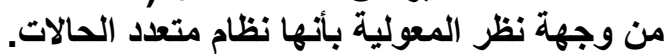

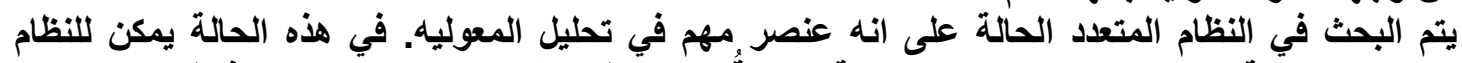

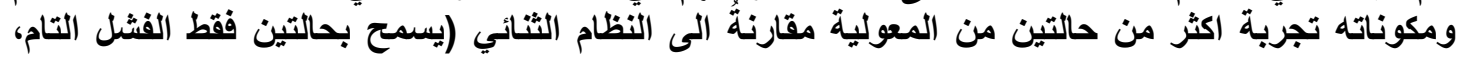
الاداء التام).

لقد تم تحسين النموذج الرياضي للنظام المتعدد الحالات نحن نفحص النظام وندرسهُ جيداً للسماح

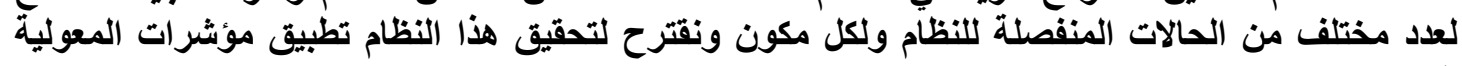




\section{المنطقية المباشرة}

هذه المؤشرات ثُقَّر التأثير الحاصل على معولية النظام المتعدد الحالة. (1,10,11,12,13

(1-1) الدالة المعولية للأنظهه المتعددة الحالات:-

\section{Reliability Function of Multi-StateSystem (MSSRF)}

ان الدالة المعولية (j) هي واحدة من افضل مقاييس المعولية للأنظمه المتعددة الحالات (MSS) المعروفة.

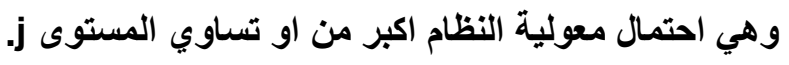

$R(j)=P_{r}\{\emptyset(x) \geq j\}, j \in\{0,1, \ldots \ldots \ldots, m-1\}$

المعادلة اعلاه لاتسحح بتحليل التغيير في معولية النظام التي يُسبيها التغير في حالات المكون.

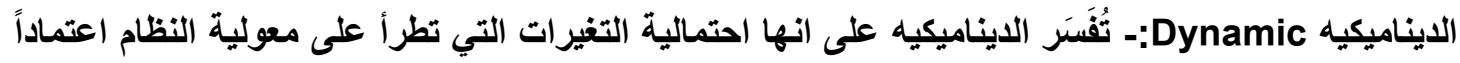

$$
\text { على حدوث تغيرات في حالة المكون. }
$$

(2-3) النظام المتعدد الحالات Multi-State Sestem:

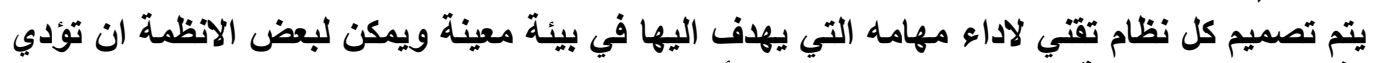

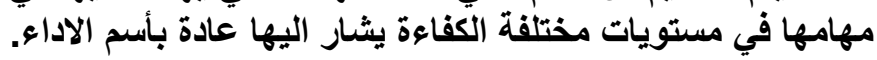

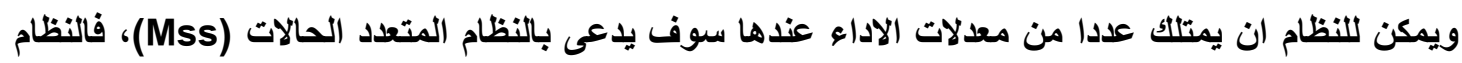
المتعدد الحالات هو نموذج رياضي يستخدم في تحليل المعولية وذلك لعرض العاد النظام مع بعض من من مستويات

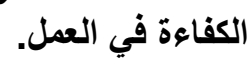

في نظام وحدة توليد يتألف من توليد ونقل الطاقه كل وحدة توليد تستطيع ان تعمل بمستويات مختلفه

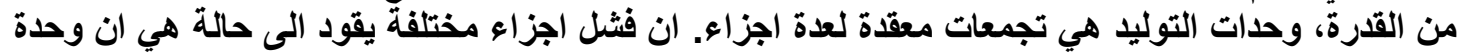

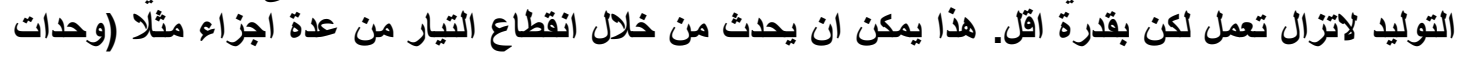

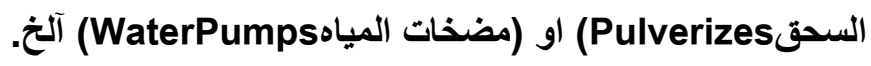

مثال على ذلك هناك وحدة توليد بقدرة 50MW معدلات الاداء (القدرة التوليديه) المقابلة لهذه الحالات

\begin{tabular}{|c|c|c|}
\hline N.of state & Generating capacity(MW) & State probability \\
\hline ا 1 عمل تام & 50 & 0.960 \\
\hline ب r عمل جزئي & 30 & 0.033 \\
\hline 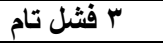 & $\mathbf{0}$ & 0.007 \\
\hline
\end{tabular}
واحتمالية الحالات متمثلة في الجدول التالي:-

عادة النظام المتعدد الحالات يتألف من عناصر او مكونات يمكن بلوررها ان تكون هي ايضا متعددة

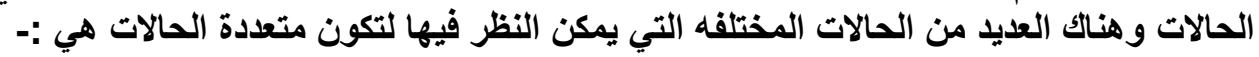

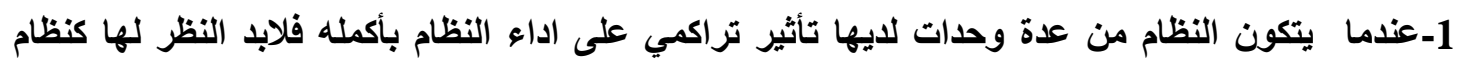

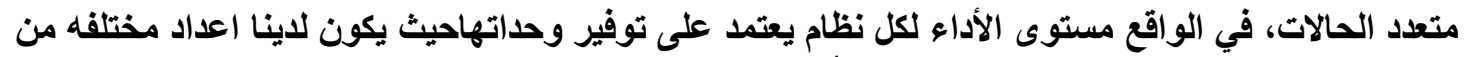

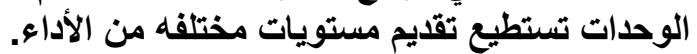
عندما تساهم وحدات مختلفه بمعدلات مختلفه من الأداء للاع للنظام التراكمي عندها يمكن لعداء من الأنظمه

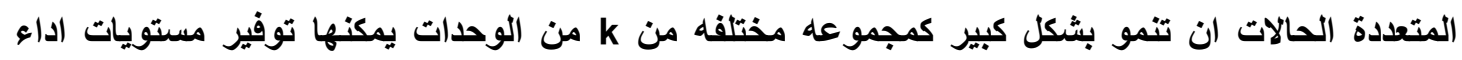
مختلفه للنظام بكامله.

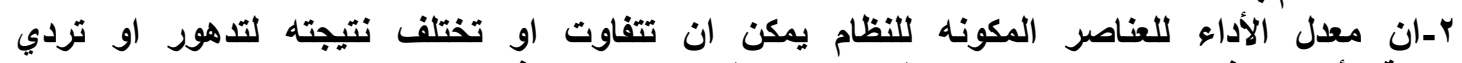

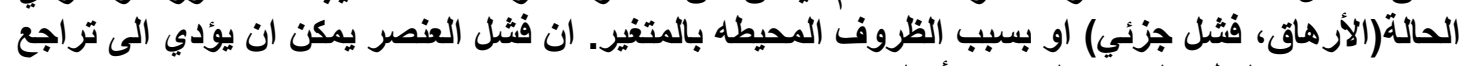
او تلهور اداء النظام المتعدد الحالات بأكملة. 


\section{المنطقية المباشرة}

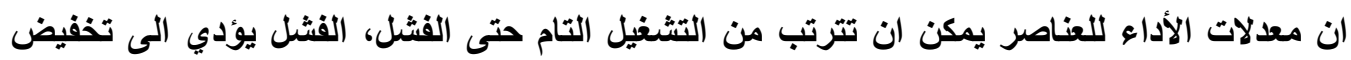

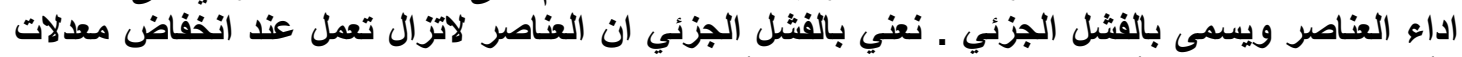

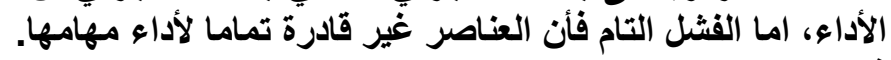

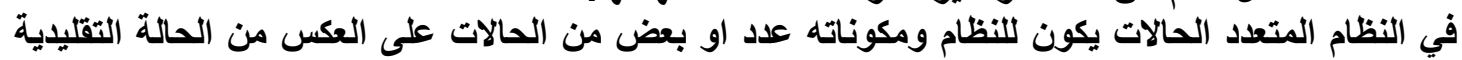

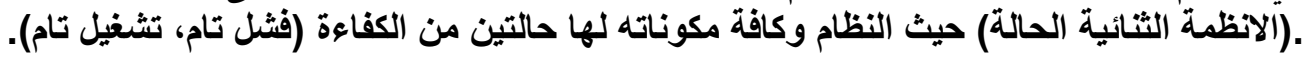

(3-3) الدالة الهيكليه للنظام المتعدد الهالات:

Structure Function of Multi-State System (SFOMSS)

الداله الهيكليه للانظمه المتعدده الحالات (mss) تستخدم لحساب (DRI) ) (مؤشرات المعوليه

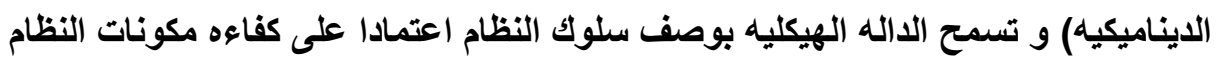

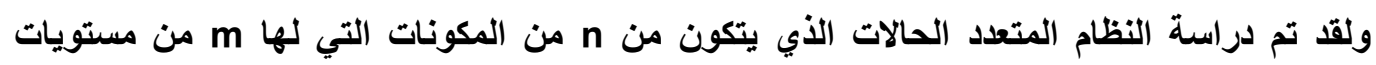

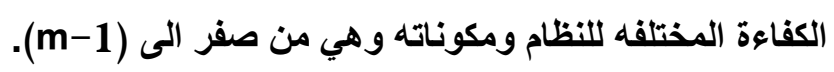

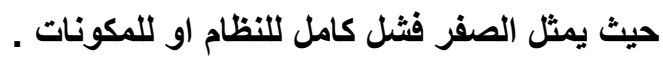

والـ (m-1) يمثل عمل كامل او تام للنظام وللمكونات وكل مكون للنظام Xئمئ يتميز بواسطة احتمالية

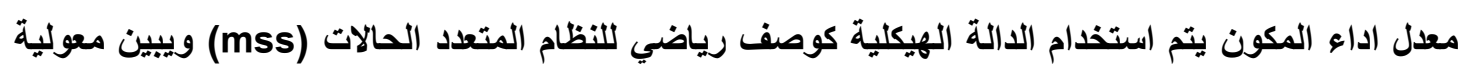
$\emptyset(x)=\left\{\begin{array}{llll}0 & \text { if } & x & \text { is failed system state } \\ s & \text { if } & x & \text { is an functioning system state }\end{array}\right.$ النظام(حالة النظام) اعتماداً على كفاءة المكونات.

Dynamic Reliability Indices (DRI)

(3-4) هؤشرات المعوليه الديناهيكية : ان مؤشرات المعولية الايناميكية يتم بواسطتها وصف التغييرات التي تحدث في معولية النظام المتعدد

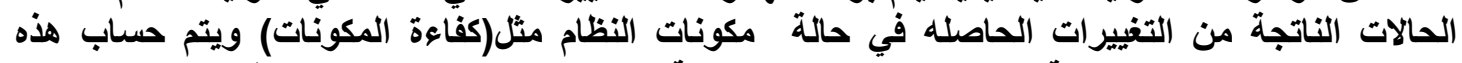

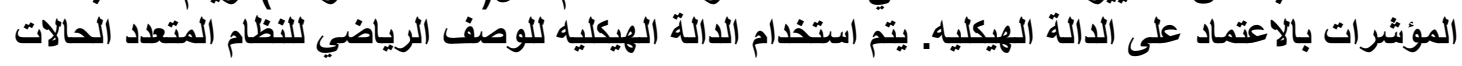
(حالة النظام) اعتمادا على كفاعة المكونات. (mss) $\Phi\left(x_{1}, \ldots x_{n}\right)=\phi(x):\left\{0, \ldots, m_{i-1}\right\}^{n} \rightarrow\{0, \ldots, m-1\}$.

$(\mathbf{i}=\mathbf{1}$

n)

Mathematical model for multi-state systems

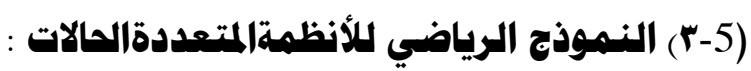
لتحليل معولية النظام المتعدد الحالات سيكون نموذج المشتقه الجزئيه المنطقيه المباشره هو النموذج الرياضي المستخدم. - nملئ

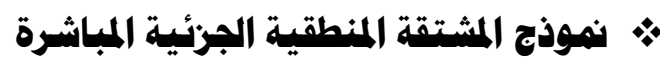

Direct partial logic derivative for mss model لتطبيق المشتقة الجزئية المنطقية المباشرة في تحليل المعولية للانظمة المتعددة الحالات نأخذ

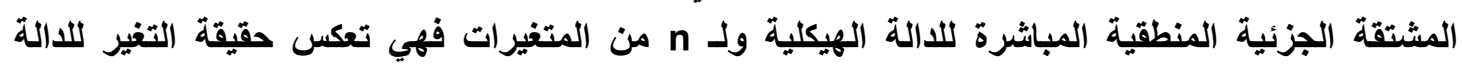

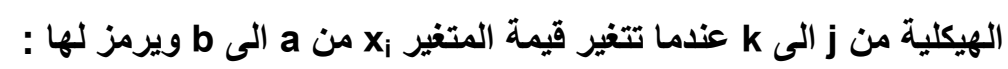
$\frac{\partial \emptyset(j \rightarrow k)}{\partial x i(a \rightarrow b]}=\left\{\begin{array}{c}m-1, \text { if }: \emptyset\left(x_{1}, \ldots, x_{i-1}, a, x_{i+1}, \ldots x_{n}\right)=j \\ \emptyset\left(x_{1, \ldots,}, x_{i+1}, \ldots, x_{n}\right)=k \\ 0, \quad 0 . w .\end{array}\right.$

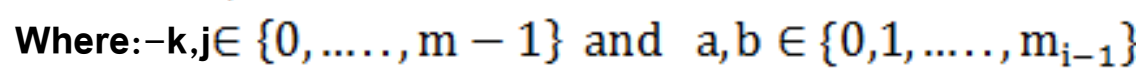




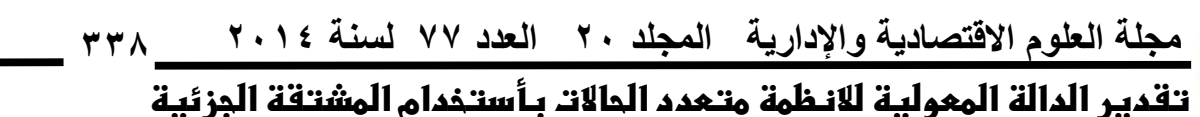

\section{المنطقية المباشرة}

(•) تدل على عملية المقارنة (التفضيل) [اي هي اما اكبر او اصغر]

$$
\frac{\partial \emptyset(\mathrm{j} \rightarrow \mathrm{k})}{\partial \mathrm{xi}(\mathrm{a} \rightarrow \mathrm{b})}=\emptyset\left(\mathrm{x}_{1}, \ldots ., \mathrm{x}_{\mathrm{i}-1}, \mathrm{a}, \mathrm{x}_{\mathrm{i}+1}, \ldots . . \mathrm{x}_{\mathrm{n}}\right) \cdot\left(\mathrm{x}_{1}, \ldots . \mathrm{x}_{\mathrm{i}-1}, \mathrm{~b}, \mathrm{x}_{\mathrm{i}+1}, \ldots . . \mathrm{x}_{\mathrm{n}}\right)
$$

اذن المشتقة الجزئية المنطقية المباشرة للدالة الهيكلية

$$
\emptyset(\mathrm{x}):\left\{0, \ldots . \mathrm{m}_{\mathrm{i}-1}\right\} * \ldots \ldots \ldots *\left\{0, \ldots \ldots \mathrm{m}_{\mathrm{n}-1}\right\} \rightarrow\{0, \ldots \ldots \mathrm{m}-1\}
$$

تسمح بدراسة تأثير تغير i-th من حالة المكونات على معولية النظام.

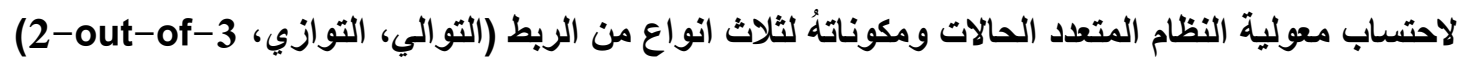
سوف نستخدم الخوارزمية التالية:ا- الخطوةالاولى: نقوم بحساب قيمة الدالة الهيكلية (x) Ø صسب خصائص النظام وطبيعة عمل هذا النظام. r- الخطوةالثانية: حساب التغير في الدالة الهيكيليه

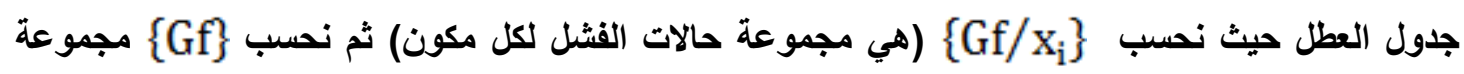
حالات الفثل للنظام حيث r- الخطوةالثالثة: حساب التغير في الدالة الهيكليه حساب

$$
\text { حيث:- للنظام. }
$$

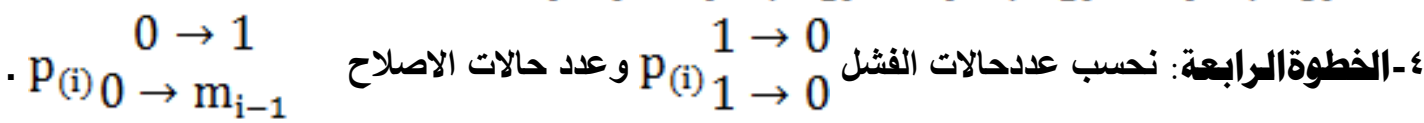

$$
\text { 0-الخطوةالخاهسة: نحسب الاحتمالية الهيكلية (i-th) من المكونات طبقا للمعادلتين: }
$$

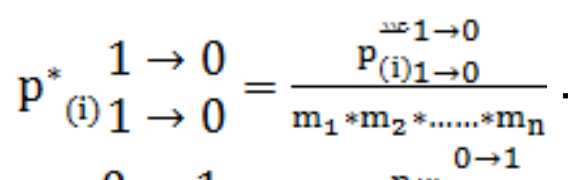

$\begin{aligned} 0 & \rightarrow 1 \\ \mathrm{P}^{*} 0 & \rightarrow \mathrm{m}_{\mathrm{i}-1}\end{aligned}=\frac{\left.\mathrm{P}_{(\mathrm{i})}\right)_{0 \rightarrow 1}^{0 \rightarrow \mathrm{m}_{\mathrm{i}-1}}}{\mathrm{~m}_{1} * \mathrm{~m}_{2} *_{\mathrm{mm}} * \mathrm{~m}_{\mathrm{n}}}$

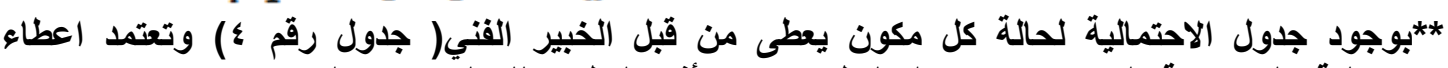

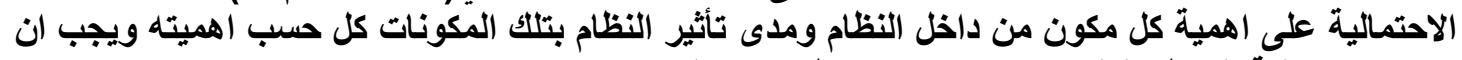

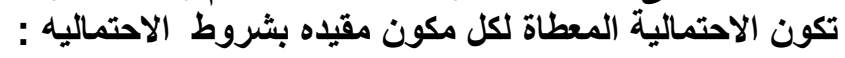
1- إن ان تكون المكونات مستقله.

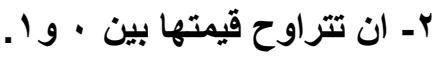

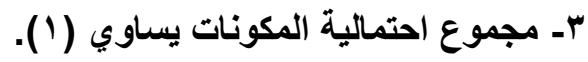
ا- الخطوة|لسادسة:الان سوف نحسب CDRI (المعولية الديناميكية لكل مكون) بواسطة المعادلتين

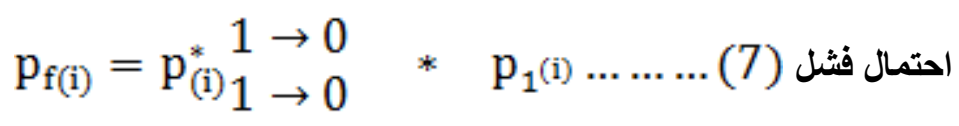




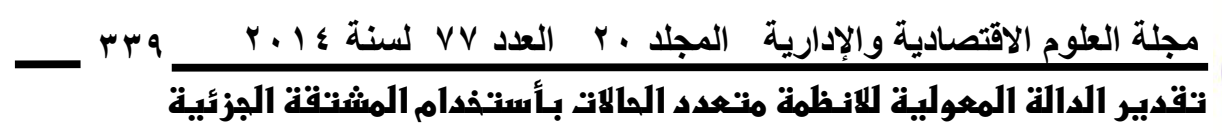

$$
\begin{aligned}
& \text { المنطقية المباشرة } \\
& p_{r(i)}=p_{(i)}^{*} \quad \begin{array}{ll}
0 \rightarrow 1 \\
0 \rightarrow m_{i-1}
\end{array} * p_{0}(i) \ldots \ldots . . . .(8) \quad \text { احتمال الاصلاح } \\
& \text { Vالفطوة السابعة: نجد اخيرا DIRI (المعولية الايناميكيةللنظام بالكامل) وحسب المعادلتين التاليتين:- } \\
& 1-\quad p_{f}=\sum_{i=1}^{n} p_{f(i)} * \underset{\substack{q \\
q \neq i}}{n}\left(1-p_{f(q)}\right) \\
& \text { 2- } \quad p_{r}=\sum_{i=1}^{n} p_{r(i)} * \prod_{\substack{q \\
q \neq 1}}^{n}\left(1-p_{f(q)}\right) \\
& \text { والمخطط التالي يوضح طريقة احتساب المعوليه الديناميكيه لكل مكون Pr(i),p } \\
& \text { - } p_{\mathrm{r}}, \mathrm{p}_{\mathrm{f}}
\end{aligned}
$$


r.

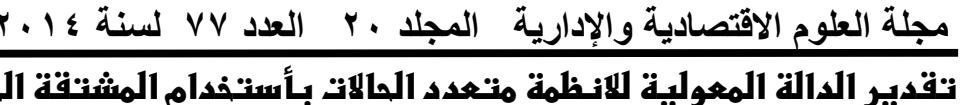

المنطقية المباشرة

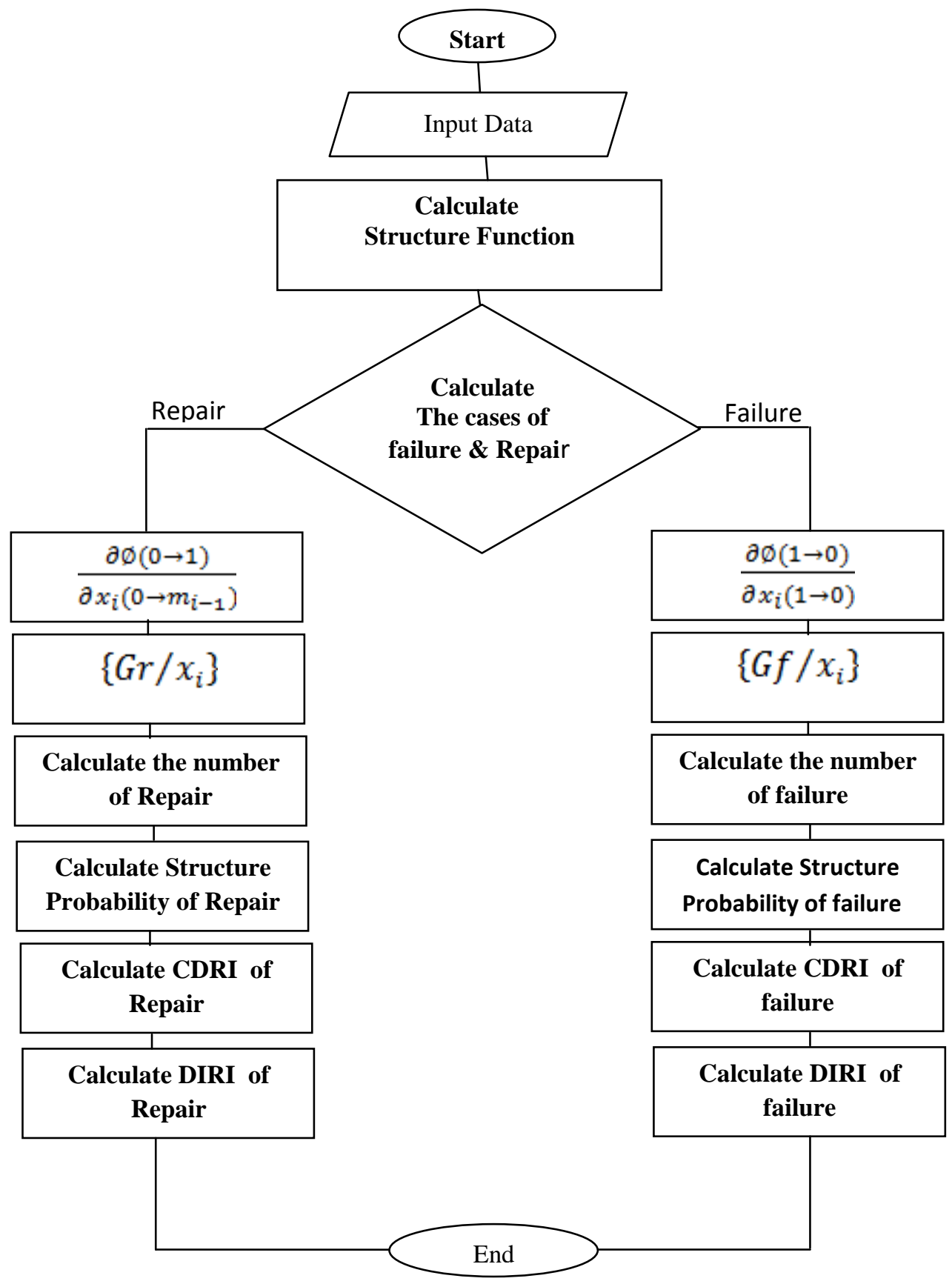

المخطط الانسيابي ( Flow Chart )

للنظام المتعدد الحالات ( MSS ) 


\section{المنطقية المباشرة}

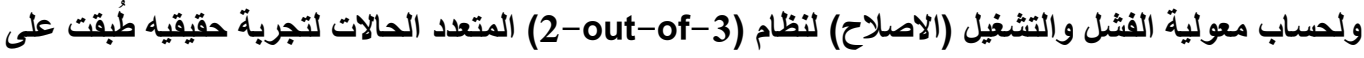

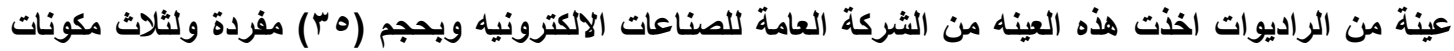

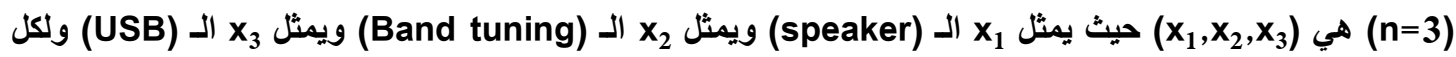
مكون من هذه المكونات ثلاث مستويات من الاداء (m=3) حيث (m)

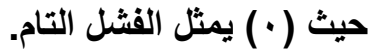

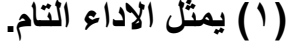

الداله الهيكليه:-

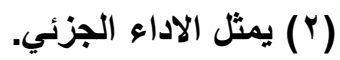

structure function

$$
\begin{gathered}
\emptyset(x)=\left\{\begin{array}{l}
0 \quad \text { if } x i \text { is failed systeam state } \\
S \quad \text { if } x i \text { is afunctioing system state }
\end{array}\right. \\
S \in\{1, \ldots \ldots, M-1\} \text { and } X_{i} \in\left\{0, \ldots \ldots, m_{i-1}\right\}
\end{gathered}
$$

\begin{tabular}{|c|c|c|c|c|}
\hline no & $\mathbf{X}_{1}$ & $\mathbf{X}_{\mathbf{2}}$ & $\mathbf{X}_{\mathbf{3}}$ & $\Phi(\mathbf{x})$ \\
\hline 1 & $\begin{array}{l}\mathbf{0} \\
\end{array}$ & $\begin{array}{l}\mathbf{0} \\
\end{array}$ & $\begin{array}{l}\mathbf{0} \\
\end{array}$ & $\begin{array}{l}\mathbf{0} \\
\end{array}$ \\
\hline 2 & $\mathbf{0}$ & 0 & $\mathbf{0}$ & 0 \\
\hline 3 & 0 & $\overline{0}$ & 0 & 0 \\
\hline 4 & $\mathbf{0}$ & $\mathbf{0}$ & 0 & 0 \\
\hline 5 & $\mathbf{0}$ & 0 & $\mathbf{0}$ & 0 \\
\hline 6 & 0 & $\mathbf{0}$ & 1 & 0 \\
\hline 7 & $\mathbf{0}$ & 2 & 0 & 0 \\
\hline 8 & $\mathbf{0}$ & 2 & 0 & $\mathbf{0}$ \\
\hline 9 & O & 1 & 0 & 0 \\
\hline 10 & $\mathbf{0}$ & 1 & $\mathbf{0}$ & $\mathbf{0}$ \\
\hline 11 & 0 & 0 & 2 & 0 \\
\hline 12 & 1 & 1 & 2 & 2 \\
\hline 13 & 1 & 0 & 1 & 1 \\
\hline 14 & 1 & O & 1 & 1 \\
\hline 15 & 1 & 0 & 1 & 1 \\
\hline 16 & 1 & 1 & 1 & 1 \\
\hline 17 & 1 & 2 & 0 & 1 \\
\hline 18 & 1 & O & 1 & 1 \\
\hline 19 & 1 & 0 & 1 & 1 \\
\hline 20 & 1 & 2 & 1 & 2 \\
\hline 21 & 1 & 1 & $\mathbf{0}$ & 1 \\
\hline 22 & 1 & 1 & 0 & 1 \\
\hline 23 & 1 & 1 & 2 & 2 \\
\hline 24 & 1 & O & 1 & 1 \\
\hline 25 & 1 & 1 & 0 & 1 \\
\hline 26 & 1 & $\mathbf{0}$ & 1 & 1 \\
\hline 27 & 1 & O & 0 & 0 \\
\hline 28 & 1 & 0 & 2 & 1 \\
\hline 29 & 2 & 2 & 1 & 2 \\
\hline 30 & 2 & 2 & 2 & 2 \\
\hline 31 & 2 & 1 & 0 & 1 \\
\hline 32 & 2 & 1 & 1 & 2 \\
\hline 33 & 2 & 1 & 1 & 2 \\
\hline 34 & 2 & 1 & 1 & 2 \\
\hline 35 & 2 & 0 & 0 & 0 \\
\hline
\end{tabular}

جدول رقم ( ') يبين حساب الداله الهيكليه لـ (2-out-of-3)(MSS)

في الجدول رقم (1) :- (0) يمثل عطل تام، (1) يمثل تثنغيل تام، (2) تثغيل جزئي. فقي حالة كانت

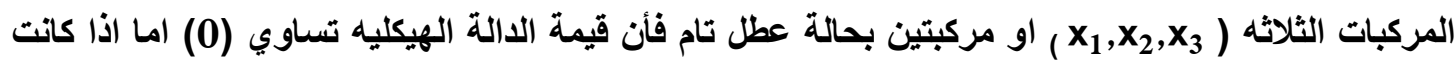




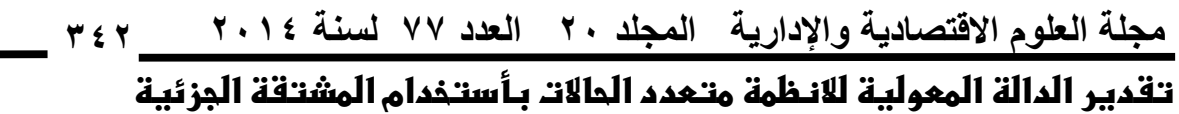

\section{المنطمقبة المباشثرة}

مركبتين بحالة تشغيل تام او جزئي او تكون الثلاث مركبات بحالة تشغيل تام فأن قيمة الداله الهيكليه تشاوي

(1) واذا كاتت المركبات الثلاثه بحالة تشغيل تام وجزئي فأن قيمة الداله الهيكليه تشاوي (2).

-

لحساب التغيير الحاصل على قيمة الداله الهيكليه في حالة عطل اي مركبه من المركبات الثلاثه نستخدم $\frac{\partial \emptyset(1 \rightarrow 0)}{\partial x_{i}(1 \rightarrow 0)}-\mathbf{l}$

جدول رقم (2) جدول العطل

\begin{tabular}{|c|c|c|c|c|c|c|}
\hline no & $\mathbf{X}_{1}$ & $\mathbf{X}_{2}$ & $\mathbf{X}_{3}$ & $\frac{\partial \emptyset(1 \rightarrow 0)}{\partial x_{1}(1 \rightarrow 0)}$ & $\frac{\partial \emptyset(1 \rightarrow 0)}{\partial x_{2}(1 \rightarrow 0)}$ & $\frac{\partial \phi(1 \rightarrow 0)}{\partial x_{3}(1 \rightarrow 0)}$ \\
\hline 1 & 0 & 0 & 0 & $\mathbf{0}$ & $\mathbf{0}$ & $\mathbf{0}$ \\
\hline 2 & $\mathbf{0}$ & $\mathbf{0}$ & $\mathbf{0}$ & $\mathbf{0}$ & $\mathbf{0}$ & $\mathbf{0}$ \\
\hline 3 & 0 & 0 & 0 & 0 & 0 & 0 \\
\hline 4 & $\mathbf{0}$ & $\mathbf{0}$ & $\mathbf{0}$ & $\mathbf{0}$ & $\mathbf{0}$ & $\mathbf{0}$ \\
\hline 5 & $\mathbf{0}$ & $\mathbf{0}$ & $\mathbf{0}$ & $\mathbf{0}$ & $\mathbf{0}$ & $\mathbf{0}$ \\
\hline 6 & $\mathbf{0}$ & $\mathbf{0}$ & 1 & $\mathbf{0}$ & $\mathbf{0}$ & $\mathbf{0}$ \\
\hline 7 & $\overline{\mathbf{0}}$ & 2 & $\mathbf{0}$ & $\mathbf{0}$ & $\overline{\mathbf{0}}$ & $\overline{\mathbf{0}}$ \\
\hline 8 & $\mathbf{0}$ & 2 & $\mathbf{0}$ & $\mathbf{0}$ & $\mathbf{0}$ & 0 \\
\hline 9 & 0 & 1 & 0 & 0 & 0 & 0 \\
\hline 10 & 0 & 1 & $\mathbf{0}$ & 0 & 0 & 0 \\
\hline 11 & 0 & 0 & 2 & 0 & 0 & 0 \\
\hline 12 & 1 & 1 & 2 & 0 & 0 & 0 \\
\hline 13 & 1 & $\mathbf{0}$ & 1 & 2 & $\mathbf{0}$ & 2 \\
\hline 14 & 1 & 0 & 1 & 2 & 0 & 2 \\
\hline 15 & 1 & $\mathbf{0}$ & 1 & 2 & $\mathbf{0}$ & 2 \\
\hline 16 & 1 & 1 & 1 & $\mathbf{0}$ & $\overline{\mathbf{0}}$ & $\overline{\mathbf{0}}$ \\
\hline 17 & 1 & 2 & $\mathbf{0}$ & 2 & 2 & 0 \\
\hline 18 & 1 & $\mathbf{0}$ & 1 & 2 & 0 & 2 \\
\hline 19 & 1 & $\mathbf{0}$ & 1 & 2 & 0 & 2 \\
\hline 20 & 1 & 2 & 1 & $\mathbf{0}$ & $\mathbf{0}$ & $\mathbf{0}$ \\
\hline 21 & 1 & 1 & $\mathbf{0}$ & 2 & 2 & $\mathbf{0}$ \\
\hline 22 & 1 & 1 & $\mathbf{0}$ & 2 & 2 & $\mathbf{0}$ \\
\hline 23 & 1 & 1 & 2 & $\mathbf{0}$ & $\mathbf{0}$ & $\mathbf{0}$ \\
\hline 24 & 1 & $\mathbf{0}$ & 1 & 2 & $\mathbf{0}$ & 2 \\
\hline 25 & 1 & 1 & $\mathbf{0}$ & 2 & 2 & $\mathbf{0}$ \\
\hline 26 & 1 & $\mathbf{0}$ & 1 & 2 & $\mathbf{0}$ & 2 \\
\hline 27 & 1 & $\overline{0}$ & $\mathbf{0}$ & 0 & $\overline{0}$ & $\mathbf{0}$ \\
\hline 28 & 1 & $\mathbf{0}$ & 2 & 2 & $\mathbf{0}$ & $\mathbf{0}$ \\
\hline 29 & 2 & 2 & 1 & $\mathbf{0}$ & $\mathbf{0}$ & $\mathbf{0}$ \\
\hline 30 & 2 & 2 & 2 & $\mathbf{0}$ & $\mathbf{0}$ & $\mathbf{0}$ \\
\hline 31 & 2 & 1 & $\mathbf{0}$ & 0 & 2 & 0 \\
\hline 32 & 2 & 1 & 1 & $\mathbf{0}$ & $\mathbf{0}$ & $\mathbf{0}$ \\
\hline 33 & 2 & 1 & 1 & 0 & 0 & 0 \\
\hline 34 & 2 & 1 & 1 & $\mathbf{0}$ & $\overline{\mathbf{0}}$ & $\mathbf{0}$ \\
\hline 35 & 2 & 0 & 0 & $\mathbf{0}$ & $\mathbf{0}$ & 0 \\
\hline
\end{tabular}




\section{المنطقية المباشرة}

في جدول العطل رقم (2) يحسب التغير في المشتقه المنطقيه الجزئيه المباشره اساس مثلا x =1 عندما تتغير حالته ولمره واحدة فقط من الحاله (1) الى الحاله (0) فأن قيمة التغير في

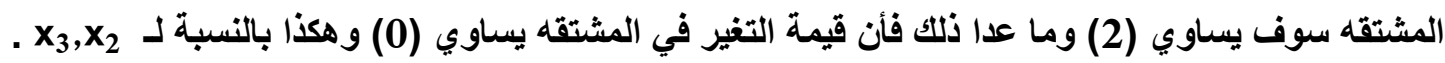

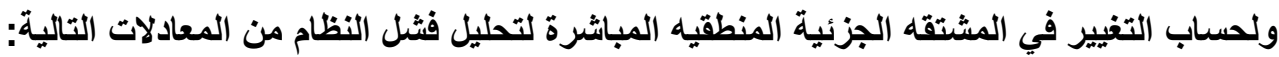
$\frac{\partial \emptyset(1 \rightarrow 0)}{\partial x_{1}(1 \rightarrow 0)}=\left\{\begin{array}{c}m-1, \emptyset\left(1, x_{2} x_{3}\right)=1> \\ \emptyset\left(0, x_{2} x_{3}\right)=0\end{array}\right.$ $\frac{\partial \emptyset(1 \rightarrow 0)}{\partial x_{2}(1 \rightarrow 0)}=\left\{\begin{array}{c}m-1, \emptyset\left(x_{1}, 1, x_{3}\right)=1> \\ \emptyset\left(x_{1}, 0, x_{3}\right)=0\end{array}\right.$ $\frac{\partial \emptyset(1 \rightarrow 0)}{\partial x_{3}(1 \rightarrow 0)}=\left\{\begin{array}{c}m-1, \emptyset\left(x_{1}, x_{2}, 1\right)=1> \\ \emptyset\left(x_{1}, x_{2}, 0\right)=0\end{array}\right.$ حالات الفشل عندما X

a-

$101,110,102,120$

b- $\quad$ عطل المركبه الثانيه (x)

110,210

c-

101

لقد تم الحصول على حالات الفشل لكل مركبه بالاعتماد على الجدول رقم (1) والعلاقات الثلاثه اعلاه. - الخطوه الثانيه: حساب جدول التشغيل.

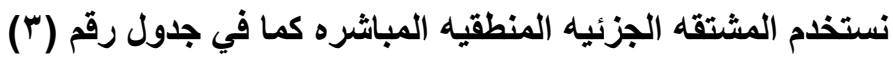

\begin{tabular}{|l|l|l|l|l|l|l|}
\hline No. & $\mathbf{x 1}$ & $\mathbf{x 2}$ & $\mathbf{x 3}$ & $\frac{\partial \emptyset(0 \rightarrow 1)}{\partial x_{1}(0 \rightarrow 2)}$ & $\frac{\partial \emptyset(0 \rightarrow 1)}{\partial x_{2}(0 \rightarrow 2)}$ & $\frac{\partial \emptyset(0 \rightarrow 1)}{\partial x_{3}(0 \rightarrow 2)}$ \\
\hline 1 & 0 & 0 & 0 & 0 & 0 & 0 \\
\hline 2 & 0 & 0 & 0 & 0 & 0 & 0 \\
\hline 3 & 0 & 0 & 0 & 0 & 0 & 0 \\
\hline 4 & 0 & 0 & 0 & 0 & 0 & 0 \\
\hline 5 & 0 & 0 & 0 & 0 & 0 & 0 \\
\hline 6 & 0 & 0 & 1 & 2 & 2 & 0 \\
\hline 7 & 0 & 2 & 0 & 2 & 0 & 2 \\
\hline 8 & 0 & 2 & 0 & 2 & 0 & 2 \\
\hline 9 & 0 & 1 & 0 & 2 & 0 & 2 \\
\hline 10 & 0 & 1 & 0 & 2 & 0 & 2 \\
\hline 11 & 0 & 0 & 2 & 2 & 2 & 0 \\
\hline 12 & 1 & 1 & 2 & 0 & 0 & 0 \\
\hline 13 & 1 & 0 & 1 & 0 & 0 & 0 \\
\hline 14 & 1 & 0 & 1 & 0 & 0 & 0 \\
\hline 15 & 1 & 0 & 1 & 0 & 0 & 0 \\
\hline 16 & 1 & 1 & 1 & 0 & 0 & 0 \\
\hline
\end{tabular}




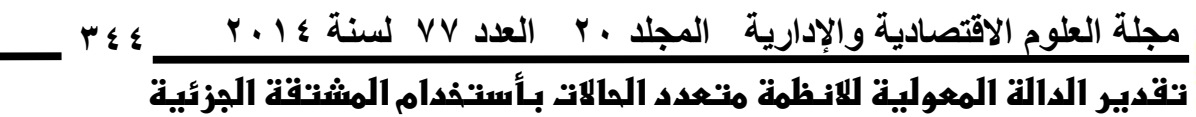

\section{المنطمقبة المبانثرة}

\begin{tabular}{|l|l|l|l|l|l|l|}
\hline 17 & 1 & 2 & 0 & 0 & 0 & 0 \\
\hline 18 & 1 & 0 & 1 & 0 & 0 & 0 \\
\hline 19 & 1 & 0 & 1 & 0 & 0 & 0 \\
\hline 20 & 1 & 2 & 1 & 0 & 0 & 0 \\
\hline 21 & 1 & 1 & 0 & 0 & 0 & 0 \\
\hline 22 & 1 & 1 & 0 & 0 & 0 & 0 \\
\hline 23 & 1 & 1 & 2 & 0 & 0 & 0 \\
\hline 24 & 1 & 0 & 1 & 0 & 0 & 0 \\
\hline 25 & 1 & 1 & 0 & 0 & 0 & 0 \\
\hline 26 & 1 & 0 & 1 & 0 & 0 & 0 \\
\hline 27 & 1 & 0 & 0 & 0 & 2 & 2 \\
\hline 28 & 1 & 0 & 2 & 0 & 0 & 0 \\
\hline 29 & 2 & 2 & 1 & 0 & 0 & 0 \\
\hline 30 & 2 & 2 & 2 & 0 & 0 & 0 \\
\hline 31 & 2 & 1 & 0 & 0 & 0 & 0 \\
\hline 32 & 2 & 1 & 1 & 0 & 0 & 0 \\
\hline 33 & 2 & 1 & 1 & 0 & 0 & 0 \\
\hline 34 & 2 & 1 & 1 & 0 & 0 & 0 \\
\hline 35 & 2 & 0 & 0 & 0 & 0 & 0 \\
\hline
\end{tabular}

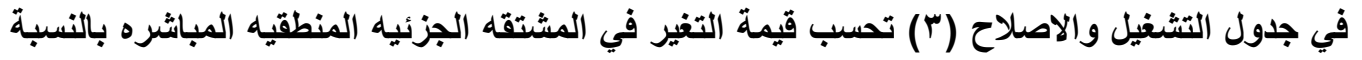

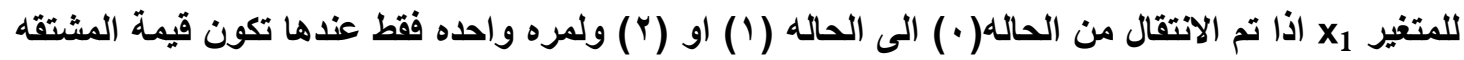

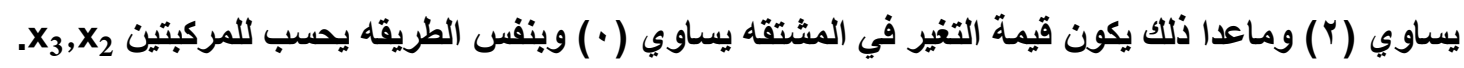
لحساب حالات التثغيل والاصلاح لكل مركبه عندما $\frac{\partial \emptyset(0 \rightarrow 1)}{\partial x_{1}(0 \rightarrow 2)}=\emptyset\left(0, x_{2}, x_{3}\right)=0<\emptyset\left(1, x_{2}, x_{3}\right)=1$

$\frac{\partial \emptyset(0 \rightarrow 1)}{\partial x_{2}(0 \rightarrow 2)}=\emptyset\left(x_{1}, 0, x_{3}\right)=0<\emptyset\left(x_{1}, 1, x_{3}\right)=1$

$\frac{\partial \emptyset(0 \rightarrow 1)}{\partial x_{3}(0 \rightarrow 2)}=\emptyset\left(x_{1}, x_{2}, 0\right)=0<\emptyset\left(x_{1}, x_{2}, 1\right)=1$

سوف تكون حالات الاصلاح عندهاهي:-

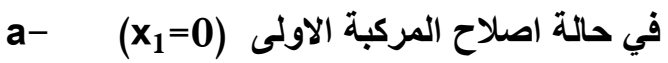

$001,020,010,002$

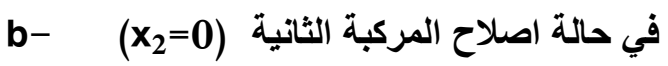

$001,002,100,200$

C- (Xي حالة|صلاح المركبة الثالثة

020,010,100,200 لقد تم الحصول على حالات الاصلاح لكل مركبه بالاعتماد على الجدول رقم (1) وعلى العلاقات الثلاثه السابقه: - - الفطوة الثالثة: وحسب الخوارزمية سوف نحسب 


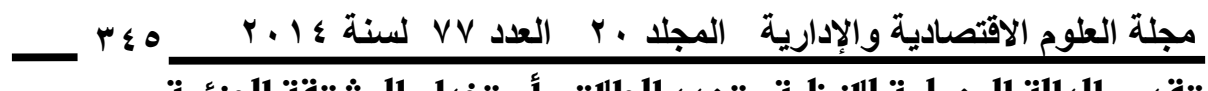

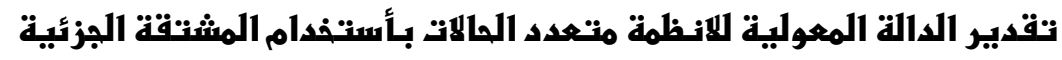

\section{المنطقية المباشرة}

$$
\begin{aligned}
1- & \left\{G f \mid x_{1}\right\}=\{101,110,102,120\} \\
2- & \left\{G f \mid x_{2}\right\}=\{110,210\} \\
\text { 3- } & \left\{G f \mid x_{3}\right\}=\{101\} \\
\therefore & \{G f\}=\{101,102,110,210,120\}
\end{aligned}
$$

وينفس الطريقة نحسب

$1-\left\{G r \mid x_{1}\right\}=\{001,020,010,002\}$

2- $\left\{G r \mid x_{2}\right\}=\{002,001,100,200\}$

3- $\quad\left\{G r \mid x_{3}\right\}=\{020,010,100,200\}$

$\therefore\{G r\}=\{001,002,010,020,100,200\}$

$$
P_{(i) 1 \rightarrow m_{i-1}} \begin{aligned}
1 \rightarrow 0 & \rightarrow 0 \\
P_{(i)} & \rightarrow 0
\end{aligned} \text { - }
$$

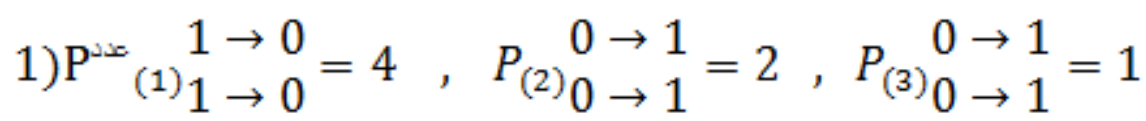

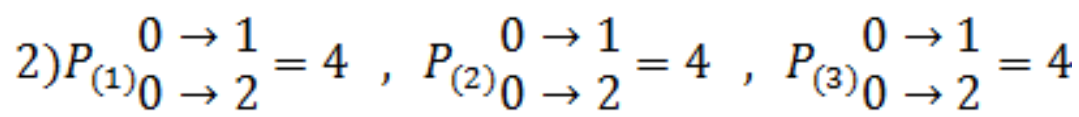

- الخطوة الخامسة: نحسب الاحتمالية|لهيكلية لـ i-th من المكونات بالاعتماد على للمعادلتين التاليتين:

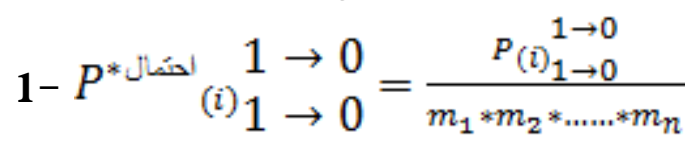

2- $P^{*} \begin{aligned} 0 & \rightarrow 1 \\ 0 & \rightarrow m_{i-1}\end{aligned}=\frac{p_{(i)_{0 \rightarrow m_{i-1}}^{0 \rightarrow 1}}}{m_{1} * m_{2} * \text { mam } * m_{n}}$

$$
\text { نطبق المعادلتين اعلاه كما يلي :- }
$$

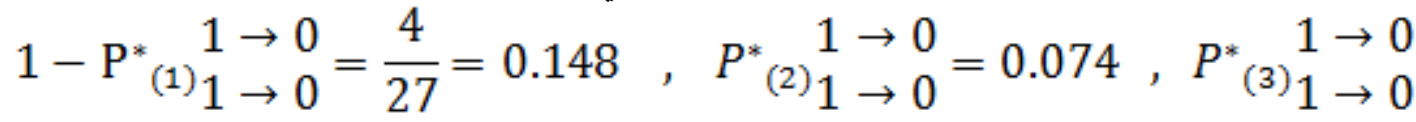
$=0.037$

$$
\begin{aligned}
& 2-P^{*} \begin{array}{r}
0 \rightarrow 1 \\
{ }^{(1)} 0 \rightarrow 2
\end{array}=\frac{4}{27}=0.148, P^{*} \begin{array}{r}
0 \rightarrow 1 \\
{ }^{(2)} 0 \rightarrow 2
\end{array}=0.148, P^{*} \begin{array}{r}
0 \\
{ }^{(3)} 0 \rightarrow 1
\end{array} \\
& =0.148
\end{aligned}
$$

* جدول رقم(ع ) القيم الاحتمالية لحالة المكونات معطى من قبل الخبير القني في المصنع : 


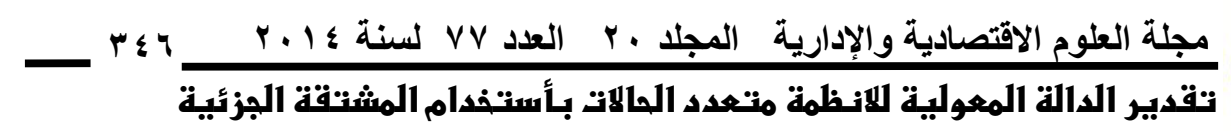

المنطقية المباشرة

\begin{tabular}{|l|l|l|l|}
\hline & 0 & 1 & 2 \\
\hline$X_{1}$ & $\ddots 1$ & $\ddots 1$ & 0.3 \\
\hline$X_{2}$ & 0.4 & 0.5 & 0.1 \\
\hline$X_{3}$ & 0.2 & 0.2 & 0.6 \\
\hline
\end{tabular}

- الخطوة السادسة: نحسب CDRI حسب المعادلتين:

$1-p_{f(i)}=p_{(i)}^{*} 1 \rightarrow 0 \quad * p_{1^{(i)}}$

$2-p_{r(i)}=p_{(i)}^{*} \quad \begin{array}{ll}1 & \rightarrow m_{i-1}\end{array} * p_{0}(i)$

$\therefore 1-P_{\mathrm{f}(1)}=(0.148)(0.6)=0.089, P_{\mathrm{f}(2)}=(0.074)(0.5)$

$=0.037, P_{\mathrm{f}(3)}=(0.037)(0.2)=0.007$

$\therefore 2-P_{\mathrm{r}(1)}=(0.148)(0.3)=0.044, P_{\mathrm{r}(2)}=(0.148)(0.1)$

$=0.015, P_{\mathrm{r}(3)}=(0 \cdot 148)(0.6)=0.089$

- الخطوة السابعة: الآن نحسب (DIRI) للنظام المتعدالحالات، حيث نقوم بتقدير فثل اواصلاح النظام $1-p_{f}=\sum_{i=1}^{n} p_{f(i)} * \quad \prod_{q-1, q \neq i}^{n}\left(1-p_{f(q)}\right)$

بأكمله بالاعتماد على المعادلتين:

2- $p_{r}=\sum_{i=1}^{n} p_{r(i)} * \quad \prod_{q-1, q \neq i}^{n}\left(1-p_{r(q)}\right)$

$p_{f(1) *}\left[\left(1-p_{f(2)}\right)\right]\left[\left(1-p_{f(3)}\right)\right]$

ا - نشب احتهال فشل النظام بتطبيق المعادلة الاولى:-

$(0.089)(1-0.037)(1-0.007)$

$(0.089)(0.963)(0.993)=0.085$

$p_{f(2) *}\left[\left(1-p_{f(1)}\right)\right]\left[\left(1-p_{f(3)}\right)\right]$

$(0.037)(0.911)(0.993)=0.033$

$p_{f(3) *}\left[\left(1-p_{f(1)}\right)\right]\left[\left(1-p_{f(2)}\right)\right]$

$(0.007)(0.911)(0.963)=0.006$

احتمال فثل النظام $P_{f}=0.085+0.033+0.006=0.125$ 


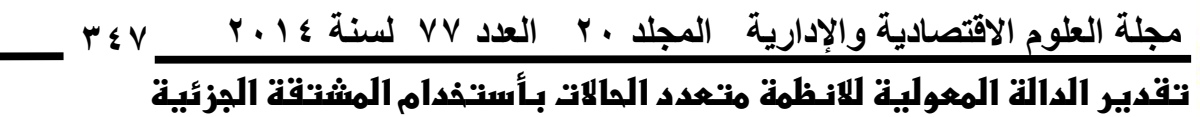

\section{المنطقية المباشرة}

$p_{r(1)} *\left[\left(1-p_{r(2))}\right)\right]\left[\left(1-p_{r(3)}\right)\right]$

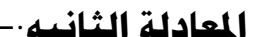

$(0.044)[1-0.015]\left[1-0.0^{\wedge}\right.$ q]

$(0.044)(0.985)(0.911)=0.0 r q$

$p_{r(2)} *\left[\left(1-p_{r(1)}\right)\right]\left[\left(1-p_{r(3)}\right)\right]$

$(0.015)(0.956)(0.911)=0.013$

$p_{r(3) *}\left[\left(1-p_{r(1)}\right)\right]\left[\left(1-p_{r(2)}\right)\right]$

$(0.089)(0.985)(0.956)=0.084$

Pr

جدول رقم(0) حساب (CDRI) للنظام (2-out-of-3)

\begin{tabular}{|c|c|c|c|c|c|c|c|c|}
\hline Comp & $\begin{aligned} 1 & \rightarrow 0 \\
(i) & 1\end{aligned}$ & $\begin{aligned} 0 & \rightarrow 1 \\
(i) & \rightarrow m_{i-1}\end{aligned}$ & $\begin{aligned} p_{(i)}^{*} & \rightarrow 0 \\
x^{k} & \rightarrow 0^{k}\end{aligned}$ & $\left\{\begin{array}{c}0 \rightarrow 1 \\
(i) 0 \rightarrow m_{i}\end{array}\right.$ & $p_{f(i)}$ & $p_{r(i)}$ & $\mathbf{P}_{\mathrm{f}}$ & $\mathbf{P}_{\mathrm{r}}$ \\
\hline $\mathbf{X}_{1}$ & 4 & $\xi$ & $\because \cdot 1 \leqslant \Lambda$ & 0.148 & 0.089 & 0.044 & \multirow{3}{*}{0.125} & \multirow{3}{*}{0.136} \\
\hline $\mathbf{X}_{2}$ & 2 & $\xi$ & 0.074 & $\because 1 \leqslant \Lambda$ & 0.037 & 0.015 & & \\
\hline $\mathbf{X}_{3}$ & 1 & 4 & 0.037 & 0.148 & 0.007 & 0.089 & & \\
\hline
\end{tabular}

- الخطوة الثاهنة: التحليل

• نلاحظ ان احتمال عطل المتغير X ان احتمال عطل المكون الثالث (2)

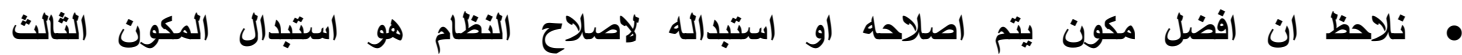
(Pr(3)=0.089) لانه يمتلك اعلى احتمال من بين المكونات لاصلاحه او استبداله. 
في هذا البحث يمكن اجمال الاستنتاجات بمايلي: 1ـ قدمنا مقياس جديد لمعولية الانظمة المتعددة الحالة الذي يحسب بواسطة الدالة الهيكلية للأنظمة المتعددة

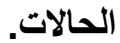

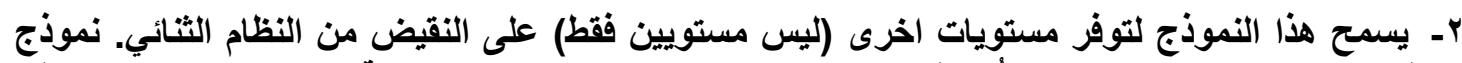

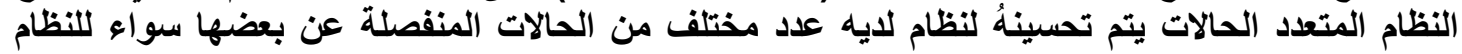

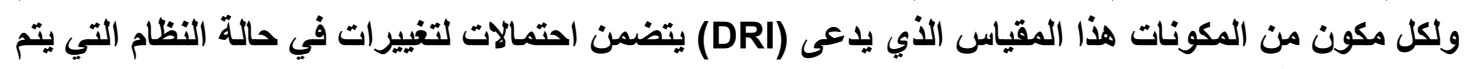

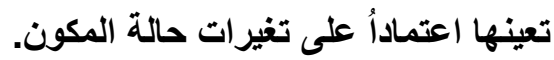
ب-يقوم الاسلوب الجديد في احتساب المعولية للأنظمة في حالة تغيرين للنظام هما فنثل النظام واصلاح

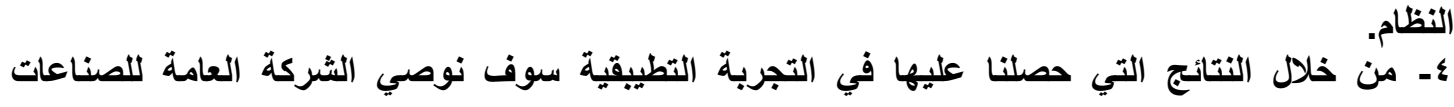

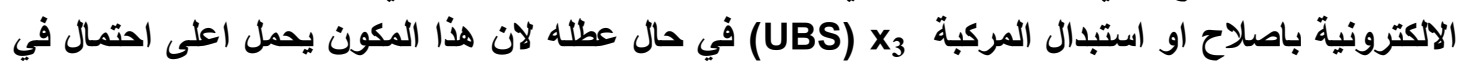

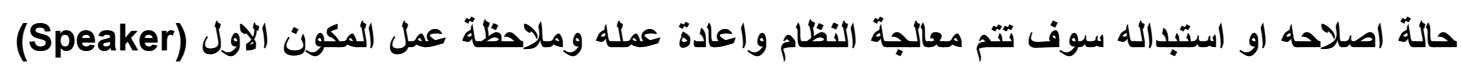
والعمل على مراعاة عدم عطله لانه في حالة عطله سوف يؤدي الى عطل النه النظام لانه يحمل اعلى احتمال الأله

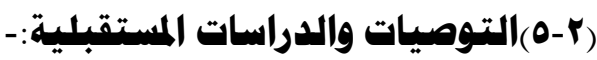

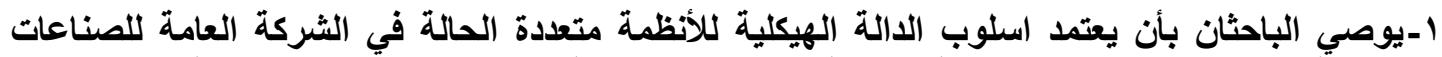

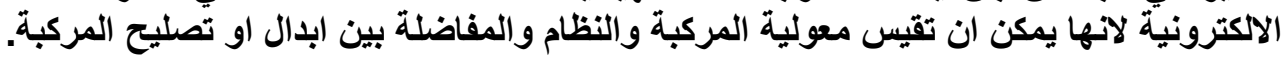

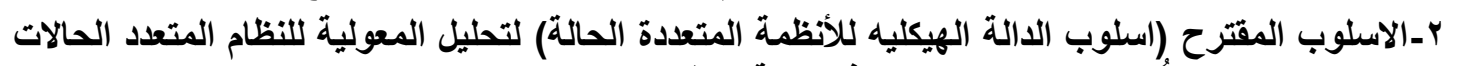

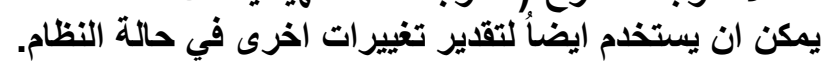

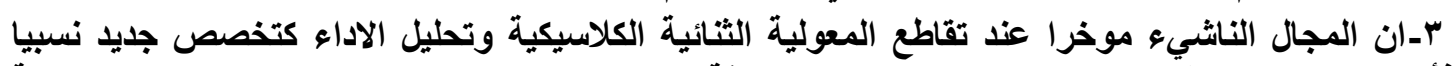

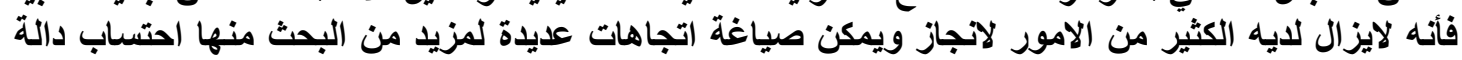

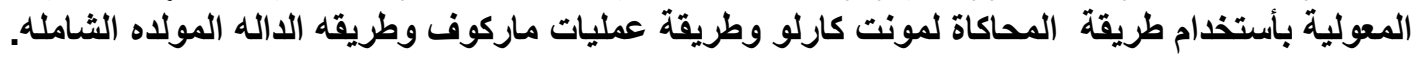

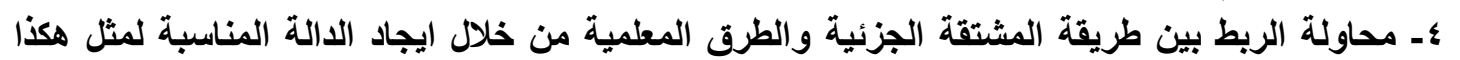


1-LISMIANSKI, A.-LEVITIN, G.: Multi-state system reliability. Assessment, Optimization and Applications. World Scientific(2003).

2-HUNG, J.-ZUO, M. J.: Multi-State k-out-of-n System and its Applications, Proc Int Symp. On Annual Reliability and Maintainability. (2000), 264-268.

3-XUE, J.-YANG,. K.: Dynamic Reliability Analysis of Coherent Multistate Systems, IEE Trinsactions on Reliability 44 No. 4 (1995), 683-688.

4-BOEDIGHEIMER, R.-KAPUR, K.: Customer-Driven Reliability Models for Multistate Coherent Systems, IEEE Transactions on Reliability 43 No. 1 (1994), 46-50.

5-ZAITSEV A, E.-LEV ASHENKO, V.: New Reliability Indices for Multi-State System, Proc $15^{\text {th }}$ IEEE European Conf on Circuit Theory and Design (2001), 345-349.

6-ZAITSEV A, E.-LEV ASHENKO, V.: Design of Dynamic Reliability Indices, Proc IEEE 32th Int Symp on Multiple-Valued Logic (2002), 144-148.

7-ZAITSEV A, E.-LEV ASHENKO, V.: New Dynamic Reliability Indices for Multi-State System, Proc $3^{\text {rd }}$ Int Conf on Mathematical Methods in Reliability: Methodology and Practice (2002), 678-690.

8-USHAKOV , I. (ed): Handbook of Reliability Engineering, Wiley, New York, (1994).

9-HUANG, J.-ZUO, M. J.: Generalized Malti-State k-out-of-n:G System, IEEE Transactions on Reliability 49 No. 1 (2000), 105-111.

10-ZAITSEV A, E.: Dyanmic Reliability Indices for Multi-State System, Journal of Dynamic System \& Geometric Theories 1 No. 2 (2003), 213-222.

11-ELENA, Z.-VITALY, L.: Reliability Analysis of Dynamic Behavior of MultiState System (2005), 1335-3632.

12- ELENA, Z.-VITALY, L.: Dyanmic Reliability Indices for Parallel, Series and k-out-of-n Multi-State System (2006), 1-4244-0008.

13- ELENA, Z.-VITALY, L.: Depending on Different Levels of System Functioning (2008), 1-4244-1543-8. 


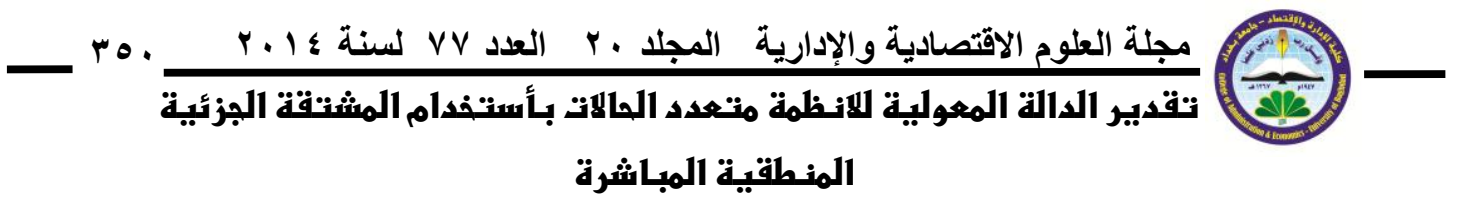

\title{
Estimation the reliability function of multi state system by using Direct Partial Logic Derivative
}

\author{
Abstract: \\ Sampeles:- \\ (MSS): Multi-state system \\ (MSSRF) : Reliability function of Multi-state system \\ $\Phi(\mathbf{x})$ : Structure function \\ (SFOMSS): Structure function of Multi-state system \\ (DRI): Dynamic Reliability Indices \\ \{Gf/Xi\}:set of failure states for each compmnent \\ \{Gr/Xi\}: set of repair states for each compmnent \\ (CDRI): Dynamic reliability for each component \\ (DIRI): Dynamic reliability for complete system
}

In this research is estimated the function of reliability dynamic of multi state systems and their compounds and for three types of systems (serial, parallel, 2out-of-3) and about two states (Failure and repair) depending on calculating the structur function allow to describing the behavior of the reliability of the system depending on the efficiency of its components. And the researcher estimates the dynamic reliability indicators which in turn describes changes in the reliability of multi state system caused by changes in the efficiency of the components system. These indicators were estimated by Direct Partial Logic derivative 